\title{
Mode Analysis of the Optical and the Microwave Waveguides Using Electromagnetic Energy Flow Lines
}

\author{
Miloš D. DavidoviĆ ${ }^{a, *}$ And Milena D. DavidoviĆ ${ }^{b}$ \\ ${ }^{a}$ School of Electrical Engineering, University of Belgrade, Serbia \\ ${ }^{b}$ Faculty of Civil Engineering, University of Belgrade, Serbia
}

\begin{abstract}
To better elucidate the essential energy transmission mechanism in the most common, typical waveguides, the electromagnetic energy flow lines for a planar optical slab waveguide and a microwave rectangular waveguide are constructed. The obtained results supply more direct and transparent insight into the distribution of electromagnetic energy in the modes of various character.
\end{abstract}

PACS numbers: 03.50.De, 42.25.Bs

\section{Introduction}

Planar optical waveguides are the key devices in construction of integrated optical circuits and semiconductor lasers. In microwave engineering practice the most common type of waveguide for high power transmission is the microwave rectangular waveguide. The analysis of the physical processes in these waveguides, which determine their behavior, is necessary for their purposeful use.

The usual and the most common approach in the analysis of optical and microwave waveguides is the use of a rigorous numerical treatment of the equations derived from Maxwell's equations and corresponding boundary conditions $[1,2]$. The numerical results are usually obtained from commercially available software (such as [3]), and it is relatively easy to produce a large amount of numerical data. Although such results are most often very precise, the drawback of this method is that it does not supply simple and transparent interpretation of the results obtained and of their physical meaning. This is especially evident if one wants to see the space energy distribution in the modes of various characters. This drawback can be diminished by construction of energy flow lines for a specific problem we are trying to solve. The method of electromagnetic (EM) flow lines has been used in [4] in the context of interpretation of two slit diffraction of a planar EM wave. EM flow lines were suggested recently in [5] as photon paths in analogy with the massive particle trajectories in Bohmian quantum mechanics.

In this paper we construct the EM energy flow lines with the help of the Poynting vector, obtained from the analytical expression for electric and magnetic fields for two cases, namely the optical slab waveguide and the microwave rectangular waveguide.

* corresponding author; e-mail: milos.d.davidovic@gmail.com

\section{EM flow lines in the planar optical slab waveguide}

Let us start with the analytical analysis of the planar optical slab waveguide. For simplicity we consider 2D problem, where there is no variation of the EM field along the $y$ axis because the width of the slab along the $y$ axis is much greater than the thickness along the $x$ axis. We also assume the surrounding medium (coating) to be infinite. The desired propagation is along the $z$ axis.

For simplicity we will only consider TM modes but the analysis is similar for other types of modes. Starting from the Maxwell equations and having in mind that there are no sources, and that there is no variation of the fields along the $y$ axis, we get simplified version of the scalar Helmholtz equation for $z$ component $\psi$ of magnetic vector potential (magnetic vector potential has only a $z$ component for TM modes [1]).

We need to satisfy both the scalar Helmholtz equation and the boundary conditions at the boundary surface between the optical core and the coating. For simplicity we assume that the constitutive parameters $\varepsilon$ and $\mu$ are homogeneous in both the core and the coating. There is also a natural boundary condition; the potential approaches zero at infinity. In the terms of the potential $\psi$ the components of the electric and magnetic fields are given by [1]:

$$
\begin{aligned}
& E_{x}=\frac{-k_{z}}{\omega \varepsilon} \frac{\partial \psi}{\partial x}, \quad E_{z}=\frac{1}{\mathrm{j} \omega \varepsilon}\left(k^{2}-k_{z}^{2}\right) \psi, \\
& H_{y}=-\frac{\partial \psi}{\partial x},
\end{aligned}
$$

where $\omega$ is the angular frequency, $k^{2}=\omega^{2} \varepsilon \mu$.

The potential for the TM field is given for the core by

$$
\psi \sim A\left\{\begin{array}{l}
\sin (u x) \\
\cos (u x)
\end{array}\right\} \mathrm{e}^{-\mathrm{j} k_{z} z} \text { for } \quad|x|<\frac{a}{2},
$$

where $a$ is the core width, and in the coating 


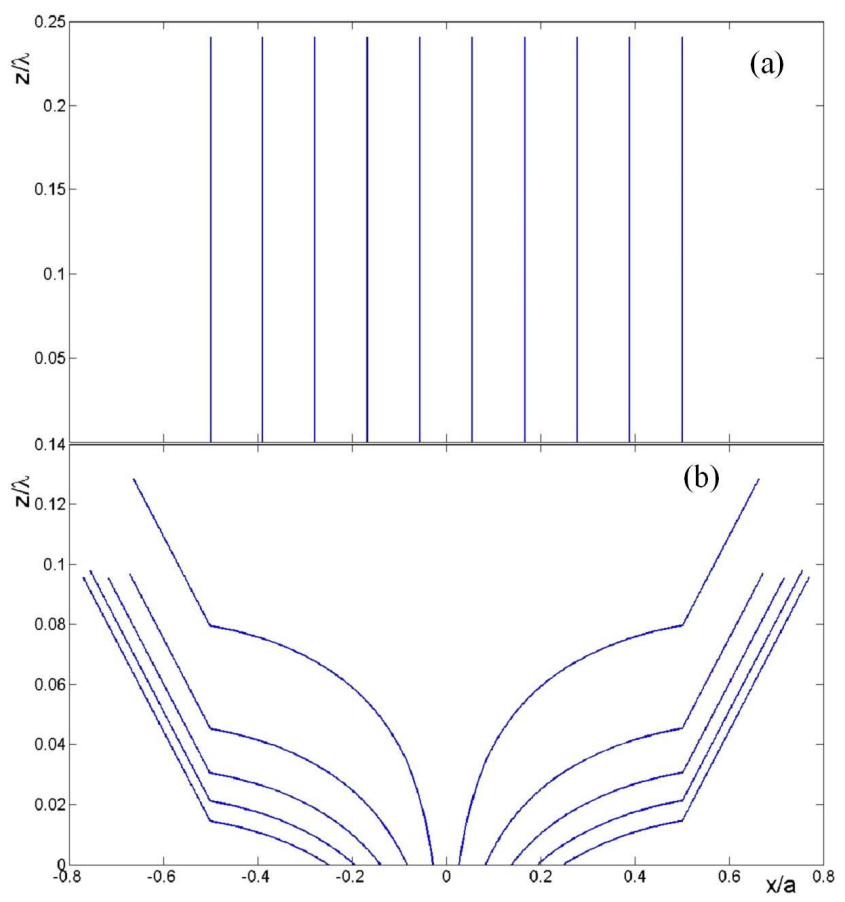

Fig. 1. Energy flow lines in optical slab waveguide for (a) propagating and (b) non-propagating modes.

$$
\psi \sim \pm B \mathrm{e}^{ \pm v x} \mathrm{e}^{ \pm \mathrm{j} k_{z} z}
$$

The values of the constants $A, B, u, v$ and $k_{z}$ are determined from the boundary conditions and separation equations. In our approximation there are two regions, the region of the core and the region of the coating. Therefore there are two separation equations

$$
\begin{aligned}
& u^{2}+k_{z}^{2}=k_{\text {core }}^{2}=\omega^{2} \varepsilon_{\text {core }} \mu_{\text {core }} \\
& -v^{2}+k_{z}^{2}=k_{\text {coating }}^{2}=\omega^{2} \varepsilon_{\text {coating }} \mu_{\text {coating. }} .
\end{aligned}
$$

The boundary conditions require that the tangential component of the EM fields is continuous at the boundary surface [1].

EM flow lines are obtained in the following way. We start by calculating the real part of the complex Poynting vector,

$$
\boldsymbol{S}=\frac{1}{2} \operatorname{Re}\left(\boldsymbol{E} \times \boldsymbol{H}^{*}\right) .
$$

From (1) and (5) we obtain

$$
\begin{aligned}
\boldsymbol{S} & =\frac{1}{2} \\
& \times \operatorname{Re}\left(\boldsymbol{e}_{x} \frac{1}{\mathrm{j} \omega \varepsilon}\left(k^{2}-k_{z}^{2}\right) \psi \frac{\partial \psi^{*}}{\partial x}+\boldsymbol{e}_{z} \frac{k_{z}}{\omega \varepsilon} \frac{\partial \psi}{\partial x} \frac{\partial \psi^{*}}{\partial x}\right) .
\end{aligned}
$$

It is apparent that the vector $\boldsymbol{S}$ does not have the component along the $y$ axis, therefore there will be no propagation of energy along the $y$ axis. For propagating modes, the values $u, v$ are real and positive and in that case $\psi \frac{\partial \psi^{*}}{\partial x}$ and $\left(k^{2}-k_{z}^{2}\right)$ are also real (for real constitutive parameters) as can be seen from Eqs. (2)-(5). In that case $\boldsymbol{S}$ does not have the component along the $x$ axis, i.e. there is no propagation of energy along the $x$ axis. So the energy flow lines will be just straight lines parallel to the $z$ axis. The solutions of above equations can also have the complex values of $u, v$ and therefore $k_{z}$. In that case the $x$ component of the Poynting vector will exist.

Now we can proceed with the construction of the energy flow lines. We start from an arbitrary point $P$ in space. In that point we calculate the vector $\boldsymbol{S}$ given by (5). Since the energy flow is along the real part of the Poynting vector, we can construct the EM flow lines by solving the equation

$$
\frac{\mathrm{d} \boldsymbol{r}}{\mathrm{d} s}=\frac{\boldsymbol{S}}{|\boldsymbol{S}|},
$$

where $\boldsymbol{r}$ is a position vector of any general point $P$ on the flow line, and $\mathrm{d} s$ is the infinitesimal element of the arc length.

Energy flow lines for propagating and non-propagating modes are shown in Fig. 1a and b, respectively. From Fig. 1a one can see that the energy flow for propagating mode is along the desired direction ( $z$ axis) while for non-propagating mode one can see the direction change at the boundary i.e. the flow lines exit from the core, meaning the energy is radiated into the coating. Let us note that in this model where the coating is infinite the non-propagating modes become radiating modes. If the coating is of finite size, then there are two types of non-propagating modes: leaky modes and radiating modes [6].

\section{EM energy flow lines in the microwave rectangular waveguide}

Energy flow lines for the microwave rectangular waveguide can be obtained in an analogous way as for the dielectric slab. Because the guiding region (dielectric) is surrounded by perfect electric conductor the expressions for the scalar potential and the fields are much simpler [1]. We consider the rectangular waveguide with dimensions $a$ and $b$ in the $x$ and $y$ directions, respectively, filled with a homogeneous dielectric, and excited with the wave propagating in the $z$ direction. If guiding region is without losses, EM flow lines are parallel to the $z$ axis as in the case of the optical slab waveguide.

If the dielectric has losses, which can be modeled by introducing the complex constitutive parameters, then the flow lines will go toward the conducting walls as shown in Fig. 2.

\section{Discussion and conclusion}

The electromagnetic energy flow lines which are derived with the help of the Poynting vector are constructed. The electric and magnetic fields are expressed in the terms of a scalar wave function [1] that explicitly takes into account the boundary conditions imposed by the structures we analyzed. 

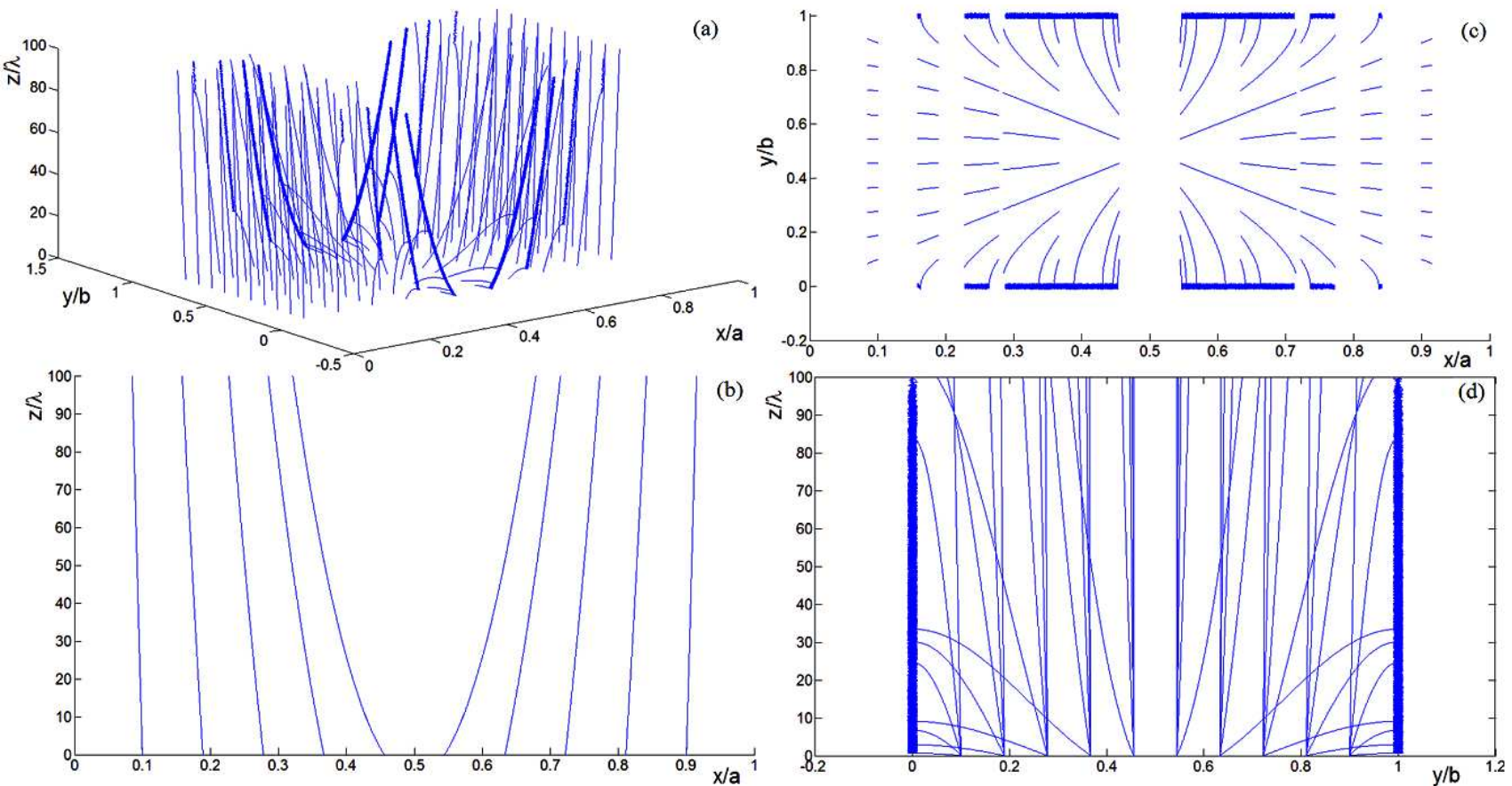

Fig. 2. EM flow lines for the rectangular microwave waveguide in case of the excitation by TE11 mode shown in different views: (a) $3 \mathrm{D}$, (b) $x y$, (c) $x z$, (d) $y z$ view.

For the analysis of an optical slab waveguide we used simplified model that allows analytical 2D analysis. In that case, the energy flow lines are situated in the $x-z$ plane, and from the patterns of flow lines one can see the difference between the propagating and non-propagating modes. The non-propagating modes radiate into the surrounding medium (flow lines go out of the slab, Fig. 1b). For the propagating modes the energy is confined in the core (flow lines are straight and go along the $z$ axis, Fig. 1a).

We also analyzed a microwave rectangular waveguide and obtained interesting patterns of flow lines for the mode propagating in the core with losses. Namely, the main reason for use of the rectangular microwave waveguides for high power transmission instead of solid or stranded wires is to avoid the skin effect. However, as our results show, the skin effect can be avoided only if the core of the waveguide is lossless. Figure 2 shows that in the case of the waveguide with lossy core the energy flow lines concentrate near the boundaries of the core therefore causing an analogon of the skin effect which is always present when the wires are used for high frequency transmission.

The developed model of the energy flow lines can be applied in wide variety of cases. This could include for example photonic band gap structures, microwave filters and antennas, power dividers etc. However for these real life examples there is rarely an analytic solution. The common way of analysis is to obtain numerical results from commercially available software (such as [3]). With modern powerful computers it is not difficult to produce large amounts of numerical data. A more difficult task, however, is to visualize the fields, to categorize the significant electromagnetic modes inside and outside the analyzed structure and to acquire some intuitive feeling for how a complex structure absorbs and scatters electromagnetic energy. The results obtained in the present paper are encouraging in the sense that they show that our method of energy flow lines could simplify this difficult task.

\section{Acknowledgments}

Miloš Davidović and Milena Davidović acknowledge the financial support from the Ministry of Science of Serbia, under the contracts 141041G and 141003, respectively.

\section{References}

[1] R.F. Harrington, Time-harmonic Electromagnetic Fields, Wiley-IEEE Press, New York 2001.

[2] K. Okamoto, Fundamentals of Optical Waveguides, Academic Press-Elsevier, Amsterdam 2006.

[3] www.wipl-d.com .

[4] R.D. Prosser, Int. J. Theor. Phys. 15, 169 (1976).

[5] M. Davidovic, A.S. Sanz, D. Arsenovic, M. Bozic, S. Miret-Artés, Phys. Scr. T 134, 014009 (2009).

[6] J. Dakin, R.G.W. Brown, Handbook of Optoelectronics, CRC Press, Boca Raton 2006. 